\title{
Screening of SARS-CoV-2 in 299 Hospitalized Children with Hemato- oncological Diseases: A Multicenter Survey in Hubei, China"
}

\author{
Song-mi $\mathrm{WANG}^{1 \dagger}$, Fang $\mathrm{TAO}^{2 \dagger}$, Yan $\mathrm{HOU}^{3 \dagger}$, Ai ZHANG ${ }^{1}$, Hao XIONG ${ }^{2}$, Jun-jie SUN ${ }^{3}$, Xiao-ping LUO ${ }^{1}$, Yan $\mathrm{HAO}^{1}$, \\ Jian-xin $\mathrm{LI}^{2}$, Qun $\mathrm{HU}^{1 \#}$, Ai-guo LIU ${ }^{1 \#}$ \\ ${ }^{I}$ Department of Pediatrics, Tongji Hospital, Tongji Medical College, Huazhong University of Science and Technology, Wuhan \\ 430030, China \\ ${ }^{2}$ Department of Pediatric Hematology \& Oncology, Wuhan Children's Hospital, Tongji Medical College, Huazhong University \\ of Science and Technology, Wuhan 430015, China \\ ${ }^{3}$ Department of Pediatrics, Xiangyang Central Hospital, Affiliated Hospital of Hubei University of Art and Science, Xiangyang \\ 441021, China
}

(C) Huazhong University of Science and Technology 2020

\begin{abstract}
Summary: The SARS-CoV-2 infection status of hospitalized children was surveyed in the department of pediatric hematology and oncology in three different hospitals of epidemic areas in Hubei, China. A cross-sectional study was performed to investigate the clinical characteristics, lung CT scan, SARS-CoV-2 nucleic acid test and serum antibodies of hospitalized children with hemato-oncological diseases from January 23 to April 24, 2020. 299 children were enrolled in this study, including 176 males (58.9\%) and 123 females (41.1\%), aged from 2 months to 16 years. 255 cases $(85.3 \%)$ received chemotherapy or other immunosuppressive therapies, and there were 44 cases $(14.7 \%)$ of other benign diseases. Nucleic acid test was performed on 258 children $(86.3 \%)$ and one case was positive. 163 cases $(54.5 \%)$ were tested for serum antibodies, and all of them were negative. Lung CT scan was performed on 247 children (82.6\%), and 107 of them showed infectious changes. Only one case $(0.33 \%)$ of COVID-19 was diagnosed in the group. The prevalence rate of COVID-19 in enrolled children with hemato-oncological diseases in Hubei was $0.33 \%$. Immunosuppressed patients are not prone to produce related antibodies. Comprehensive protective measures and ward management can reduce the risk of SARS-CoV-2 infection in the group patients.
\end{abstract}

Key words: children; COVID-19; hemato-oncological diseases; SARS-CoV-2

In December 2019, some cases of pneumonia caused by an unknown pathogen were found in Wuhan, the capital city of Hubei, which is located in central China ${ }^{[1]}$. After active etiological investigation, on January 7,2020 , the causative pathogen was identified as a novel coronavirus. On February 11, 2020, the International Committee on Virus Classification announced that this type of virus was officially named severe acute respiratory syndrome coronavirus 2 (SARS$\mathrm{CoV}-2$ ), and on the same day WHO announced that the official name of the disease caused by this virus was

Song-mi WANG, E-mail: songmi201275955@126.com; Fang TAO, E-mail: 2453037665@qq.com; Yan HOU, E-mail: julishi@163.com

${ }^{\dagger}$ The authors contributed equally to this work.

${ }^{\#}$ Corresponding authors, Qun HU, E-mail: qunhu2013@163. com; Ai-guo LIU, E-mail: drliuaiguo@163.com

${ }^{*}$ This project was supported by Huazhong University of Science and Technology Emergency Technology Research Project Response to COVID-19 (No.2020kfyXGYJ020) and Clinical Study of the Pediatric Patients with 2019-nCOV (No. XXGZBDYJ005).
COVID-19. The proportion of children in COVID-19 patients is significantly lower than that in adults. The literature has reported the clinical characteristics and outcomes of the population with a history of tumors in adult COVID-19 patients $^{[2,3]}$. However, there are few reports on COVID-19 in pediatric cancer patients. In order to investigate the prevalence rate of COVID-19 in pediatric hemato-oncological patients in the epidemic centers of this outbreak, we selected three representative pediatric hematological cancer centers, which were the Pediatric Hematology and Oncology Department of Wuhan Tongji Hospital, Wuhan Children's Hospital, and Xiangyang Central Hospital. Then we conducted a relevant survey of SARS-CoV-2 infection of the hospitalized children in the Department of Pediatric Hemato-oncology of the three hospitals in Hubei Province.

\section{MATERIALS AND METHODS}

\subsection{Study Design and Participants}

A cross-sectional study was performed to inves- 
tigate the SARS-CoV-2 infection status of children with hemato-oncological diseases hospitalized in three medical institutions from January 23 to April 24, 2020.

\subsection{Study Methods}

The clinical data of all enrolled children were collected, including general information, diagnosis, high-resolution CT examination results of the lungs, and whether serious clinical events occurred. SARS-CoV-2 serological antibodies (IgG and $\operatorname{IgM}$ ) were detected by magnetic particle chemiluminescence. Nasal swabs, pharyngeal swabs, and anal swabs were collected to detect SARS-CoV-2 nucleic acid by reverse transcriptionpolymerase chain reaction (RT-PCR). COVID-19 was diagnosed based on the 5th trial of the New Coronavirus Pneumonia Diagnosis and Treatment Program issued by the Health Commission of China. Serious clinical events were defined as conditions requiring admission to pediatric intensive care unit (PICU).

\subsection{Statistical Analysis}

The COVID-19 prevalence rate was calculated as the number of all patients diagnosed in the time period/ the number of all enrolled patients in this period.

\section{RESULTS}

\subsection{General Clinical Characteristics}

A total of 299 children were enrolled, including 176 males (58.9\%) and 123 females (41.1\%), aged from 2 months to 16 years, with a median age of 5 years and 2 months old. The distribution of primary diseases were as follows: leukemia $(n=160,53.5 \%)$, solid tumors $(n=31,10.4 \%)$, aplastic anemia $(n=21,7.0 \%)$, lymphoma ( $\mathrm{n}=18,6.0 \%)$, Langerhans cell histiocytosis $(n=14,4.7 \%)$, hemophagocytic syndrome $(n=6,2.0 \%)$, myelodysplastic syndrome $(n=5,1.7 \%)$, other nonneoplastic diseases $(n=44,14.7 \%) .255$ cases $(85.3 \%)$ received chemotherapy or immunosuppressive agents. Four patients (1.3\%) had a history of close contact with COVID-19 patients (table 1).

\subsection{Laboratory and Radiologic Findings}

The number of children who completed the nucleic acid test was $258(86.3 \%)$, including 257 negative cases $(99.6 \%)$, and 1 positive case $(0.4 \%)$. There were 50 patients (19.4\%) with multi-site testing, and 208 patients $(80.6 \%)$ with single-site testing. 163 cases $(54.5 \%)$ were tested for serum antibodies $(\mathrm{IgG}$ and (gM), and all were negative.

Of 247 children $(82.6 \%)$ receiving high-resolution CT examination of the lungs, 107 (43.3\%) showed infectious changes: patchy shadows in 48 patients, residual fiber strips in 21 cases, ground glass opacity in 11 cases, consolidation in 3 cases, diffused interstitial lesions in 2 cases, others (thickening of the pleura, pleural effusion, atelectasis, etc.) in 22 cases. Among the 107 children with abnormal imaging, 24 had fever symptoms, 18 had cough symptoms, and 3 had diarrhea symptoms (table 2).

\subsection{Prevalence Rate of COVID-19 and Clinical Analysis of Child with COVID-19}

Only one case of COVID-19 was diagnosed in the group. The prevalence rate of COVID-19 in enrolled children with hemato-oncological diseases was $0.33 \%$.

The COVID-19 child was an 8-year-old boy who had lived in Wuhan for a long time with his parents. He was diagnosed with acute lymphoblastic leukemia (T-lineage, high risk) more than 1 year ago. After more than one year of systemic chemotherapy, he obtained complete remission in his bone marrow and his treatment was in maintenance stage. The child was admitted to hospital without any clinical symptoms, then he received the maintenance phase

\begin{tabular}{lc}
\multicolumn{2}{c}{ Table 1 Clinical characteristics of patients } \\
\hline Characteristics & Number (\%) \\
\hline Gender & $176(58.9 \%)$ \\
Male & $123(41.1 \%)$ \\
Female & \\
Type of primary disease & $160(53.5 \%)$ \\
Leukemia & $31(10.4 \%)$ \\
Solid tumor & $21(7.0 \%)$ \\
Aplastic anemia & $18(6.0 \%)$ \\
Lymphoma & $14(4.7 \%)$ \\
Langerhans cell histiocytosis & $6(2.0 \%)$ \\
Hemophagocytic syndrome & $5(1.7 \%)$ \\
Myelodysplastic syndrome & $44(14.7 \%)$ \\
Other non-neoplastic diseases & \\
Use of chemotherapy or immunosuppressants & $255(85.3 \%)$ \\
Yes & $44(14.7 \%)$ \\
No & \\
Symptoms & $45(15.0 \%)$ \\
Fever & $23(7.7 \%)$ \\
Cough & $3(1.0 \%)$ \\
Diarrhea & \\
Agranulocytosis & $106(35.5 \%)$ \\
Yes & $193(64.5 \%)$ \\
No & \\
Close contact with COVID-19 patients & $4(1.3 \%)$ \\
Yes & $295(98.7 \%)$ \\
No &
\end{tabular}

Table 2 Results of examinations of COVID-19

\begin{tabular}{lc}
\hline Characteristics & Number (\%) \\
\hline Chest CT & $140(56.7 \%)$ \\
Normal & $107(43.3 \%)$ \\
Abnormal & 48 \\
Patchy shadows & 21 \\
Residual fiber strips & 11 \\
Ground-glass opacities & 3 \\
Consolidation & 2 \\
Diffused interstitial lesions & 22 \\
Others & \\
Nucleic acid testing of SARS-CoV-2 & $257(99.6 \%)$ \\
Negative & $1(0.4 \%)$ \\
Positive & \\
IgM and IgG antibody detection of SARS-CoV-2 & \\
Negative & $163(100 \%)$ \\
Positive & $0(0 \%)$ \\
\hline
\end{tabular}


CA regimen chemotherapy (cyclophosphamide 300 $\mathrm{mg} / \mathrm{m}^{2} /$ day+Cytarabine $300 \mathrm{mg} / \mathrm{m}^{2} /$ day). On day 6 after chemotherapy, his myelosuppressive phase was coming with neutropenia (a neutrophil count of $0.35 \times 10^{9} / \mathrm{L}$ ), lymphopenia (a lymphocyte count of $0.18 \times 10^{9} / \mathrm{L}$ ), moderate anemia (hemoglobin $67 \mathrm{~g} / \mathrm{L}$ ), and thrombocytopenia (a platelet count of $14 \times 10^{9} / \mathrm{L}$ ). Two days later, he began to have symptoms of low fever and cough. Physical examination showed only pharyngeal congestion without other positive signs. CT scan of the lungs showed consolidation of the left lower lobe and thickening of the local pleura in the right lung. Only influenza virus A antigen was positive for multiple pathogenic examinations. Despite receiving strong antiinfection and immune support treatment, the child still suffered repeated high fever and paroxysmal dry cough. Re-scan of lung CT on day 10 after fever showed patchy flocculent increased density shadow and a small amount of pleural effusion. SARS-CoV-2 nucleic acid test was performed on the day 12 after fever and the result was positive. The child was immediately transferred to the negative pressure isolation ward. All contact personnel performed COVID-19 screening and received medical observation for 2 weeks. The screening results were normal. On the 23rd day of the disease course, the child developed respiratory failure and was transferred to the PICU for treatment. During the course of the disease, multiple pharyngeal swabs were positive for nucleic acid, and antibodies remained negative. The patient's clinical classification was severe. At last his pulmonary infection was recovered after comprehensive treatment, and then he was discharged.

\subsection{Treatment and Complications}

Four cases had serious clinical events, manifesting as severe pneumonia and acute respiratory distress syndrome (ARDS). The etiological evidence showed that there was one case of SARS-CoV-2 infection, two cases of invasive fungal infection, and no pathogen was found in the last one. Including the COVID-19 case, three of them received tracheal intubation mechanical ventilation. The last one complicated with acute heart failure received non-invasive ventilation assistance. All 4 patients had recovered from pulmonary infection (table 3).

Table 3 Treatment outcomes of patients

\begin{tabular}{lc}
\hline Treatment outcomes & Number $(\%)$ \\
\hline Nosocomial infection & \\
Yes & $27(9.0 \%)$ \\
No & $272(91.0 \%)$ \\
Serious clinical events & \\
Yes & $4(1.3 \%)$ \\
No & $295(8.7 \%)$ \\
\hline
\end{tabular}

\section{DISCUSSION}

COVID-19 has rapidly escalated into a global pandemic. The epidemiological characteristics of COVID-19 reported by Chinese Center for Disease Control and Prevention showed that the proportion of COVID-19 patients under 20 years of old reported in mainland China was $2.1 \%{ }^{[4]}$.

The proportion of patients under 18 years of old reported by the US CDC was $1.7 \%^{[5]}$. Compared with adults, most pediatric patients generally had less severe infection, and a few cases were completely asymptomatic. They often recovered 1 to 2 weeks after onset and had a good prognosis ${ }^{[6]}$. SARS$\mathrm{CoV}-2$ is mainly transmitted by respiratory droplets and close contact ${ }^{[7]}$. Close contact with COVID-19 infected individuals is the main route of transmission in children ${ }^{[8]}$. In this study, only one patient was diagnosed. The patient denied the history of close contact with SARS-CoV-2 within 2 weeks. The family members of the child, the patients who stayed in the same period, and the medical staff had normal COVID-19 examinations. Therefore, it was speculated that the patient may be in the incubation period at admission.

Children with hematological malignancies are immunosuppressed due to their own disease and related treatments, which are a highly vulnerable group for SARS-CoV-2 infection. Studies have found that viral infections, including other human coronaviruses, are associated with increased morbidity and mortality of immunodeficient children ${ }^{[9]}$. Recently, it has been reported that among COVID-19 patients, the proportion of patients with tumors reached $1 \%{ }^{[2]}$. The findings of this study showed that the SARS-CoV-2 infection rate in enrolled children with hematological malignancies was $0.33 \%$. It may be related to the following reasons: on one hand, children and their families have a strong protective awareness, such as strict home isolation, maintaining safe social distance in public places, and wearing masks for a long time; on the other hand, the hospital has a strict control of nosocomial infection, such as strict COVID-19 screening for admitted children and attendants (positive diversion to designated hospitals for treatment, negative admission arranged to buffer ward for single isolation), active prevention and treatment of agranulocytosis and other measures.

Immunodeficient children are prone to develop into severe cases after being infected with SARSCoV-2. Zhang ${ }^{[3]}$ reported that $53.6 \%$ of COVID-19infected cancer patients had serious clinical events, with a mortality rate of $28.6 \%$. The child with COVID-19 in this study was clinically classified as severe. On the 23rd day of the disease course, the child suffered a serious clinical event. His SARS-CoV-2 nucleic acid test had been persistently positive for more than 2 months, along with persistently negative SARS-CoV-2 antibodies (IgM and $\operatorname{IgG}$ ) for more than 2 weeks, 
which may be related to the weak ability to clear the virus and the inability to produce antibodies due to humoral immunosuppression. Therefore, for patients with hematological malignancies, the possibility of COVID-19 cannot be ruled out by negative antibody detection, which needs to be combined with multiple nucleic acid test, epidemiological history, and lung imaging to assist in the diagnosis.

Chest CT imaging can be used to investigate pulmonary infection, but COVID-19 cannot be diagnosed only with typical CT features. A multicenter study on clinical and imaging lesions of COVID-19 showed that the typical pulmonary imaging lesions of COVID-19 patients were ground glass lesions (86.1\%) or ground-glass lesions combined with consolidation $(64.4 \%)^{[10]}$. The proportion of abnormal pulmonary CT findings in this study suggested that pulmonary infection was common in children with hematological malignancies, which is consistent with previous imaging studies of infection in children and adolescents with cancer reported by Sue et a $\left[^{[11]}\right.$. Among 107 patients with abnormal lung imaging, most of them had no such COVID-19 similar symptoms, and it is likely that some of them are not fully recovered from the pneumonia caused by chemotherapy. Fourteen cases showed typical ground-glass lesions and consolidation in pulmonary high-resolution CT. However, only one case was finally diagnosed as COVID-19. The rest 13 children had negative multiple nucleic acid test and antibody test, of which 4 cases of severe pneumonia had pathogen detections: 1 case of COVID-19, 2 cases of invasive pulmonary fungal infection, and 1 case of unknown pathogen. Their clinical symptoms and pulmonary imaging were significantly improved after targeted treatment. Lung imaging lesions in immunodeficiency children were not specific, and a variety of pathogens can lead to similar imaging changes of viral pneumonia. Therefore, multiple etiological examinations should be recommended to assist in the diagnosis.

Multiple multi-site nucleic acid tests are a better choice for SARS-CoV-2 screening in children with hematological malignancies. In this study, 258 children underwent SARS-CoV-2 nucleic acid detection, and some patients underwent multiple and multi-site nucleic acid detection to reduce the false negative rate. In the early stage of COVID-19, the positive rate of qualified respiratory samples was higher, and the positive rate of anal swab or stool samples was significantly higher than that of respiratory samples in the recovery stage of the disease $\mathrm{e}^{[12]}$. For children with thrombocytopenia, it is recommended to collect throat swab samples to reduce the damage and bleeding to the nasal mucosa, while collecting anal swab samples to assist in the diagnosis.

During the current pandemic, taking effective protective measures is still the best choice to reduce the risk of SARS-CoV-2 infection. On one hand, patients and their companions should take effective personal protection, such as hand hygiene, maintaining social distance in public places, and wearing masks correctly. On the other hand, hospitals and medical staffs need to do a good job in the following measures: the evaluation of chemotherapy patients, strict screening of COVID-19 for children and attendants before admission and ward management.

\section{Conflict of Interest Statement}

The authors declare that they have no conflict of interest.

\section{REFERENCES}

1 Huang C, Wang Y, Li X, et al. Clinical features of patients infected with 2019 novel coronavirus in Wuhan, China. Lancet, 2020,395(10223):497-506

2 Liang W, Guan W, Chen R, et al. Cancer patients in SARS-CoV-2 infection: a nationwide analysis in China. Lancet Oncol, 2020,21(3):335-337

3 Zhang L, Zhu F, Xie L, et al. Clinical characteristics of COVID-19-infected cancer patients: a retrospective case study in three hospitals within Wuhan, China. Ann Oncol, 2020,31(7):894-901

4 Epidemiology Working Group for NCIP Epidemic Response. The epidemiological characteristics of an outbreak of 2019 novel coronavirus diseases (COVID-19) in China. Chin J Epidemiol (Chinese), 2020,41(2):145-151

5 CDC COVID-19 Response Team, Coronavirus Disease 2019 in Children - United States, February 12 - April 2, 2020. NMWR Morb Mortal Wkly Rep, 2020,69(14): 422-426

6 Dong Y, Mo X, Hu Y, et al. Epidemiological characteristics of 2143 pediatric patients with 2019 coronavirus disease in China. Pediatrics, 2020. doi: 10.1542/peds.2020-0702

7 Zhu N, Zhang D, Wang W, et al. A novel coronavirus from patients with pneumonia in China, 2019. N Engl J Med, 2020,382(8):727-733

8 Shen K, Yang, Y. Diagnosis and treatment of 2019 novel coronavirus infection in children: a pressing issue. World J Pediatr, 2020,16(3):219-221

9 Ogimi C, Englund JA, Bradford MC, et al. Characteristics and outcomes of coronavirus infection in children: the role of viral factors and an immunocompromised state. J Pediatric Infect Dis, Soc 2019,8(1):21-28

10 Zhao W, Zhong Z, Xie XZ, et al. Relation Between Chest CT Findings and Clinical Conditions of Coronavirus Disease (COVID-19) Pneumonia: A Multicenter Study. AJR Am J Roentgenol, 2020,214(5):1072-1077

11 Sue C, Kaste DO. Infection Imaging of Children and Adolescents Undergoing Cancer Therapy: A Review of Modalities and an Organ System Approach. Semin Pediatr Infect Dis, 2000,11(2): 122-141

$12 \mathrm{Xu} \mathrm{Y,} \mathrm{Li} \mathrm{XF,} \mathrm{Zhu} \mathrm{B.} \mathrm{Characteristics} \mathrm{of} \mathrm{pediatric} \mathrm{SARS-}$ $\mathrm{CoV}-2$ infection and potential evidence for persistent fecal viral shedding. Nat Med, 2020,26(4): 502-505

(Received Jun. 3, 2020; accepted July 3, 2020) 